\title{
Diástase dos músculos retoabdominais no puerpério imediato de primíparas e multíparas após o parto vaginal
}

\author{
Diastasis of rectus abdominis muscle immediately postpartum of primiparous and \\ multiparous after vaginal delivery \\ Mariana Tirolli Rett ${ }^{1}$, Fabiane Ramos de Araújo², Isabela Rocha², Rosemery Augusta da Silva²
}

\begin{abstract}
RESUMO I O presente estudo comparou a diástase dos músculos retoabdominais (DMRA) supra-umbilical (SU) e infra-umbilical (IU) entre primíparas e multíparas, correlacionou com a paridade, idade materna, índice de massa corporal (IMC) e tempo de trabalho de parto (TTP). Foram incluídas 100 primíparas com idade de 21,0 $\pm 4,4$ anos e 100 multíparas com idade de $27,2 \pm 6,2$ anos submetidas ao parto vaginal. A DMRA foi avaliada nos pontos $4,5 \mathrm{~cm}$ acima e abaixo da cicatriz umbilical com o paquímetro. Foram empregados o test $t$ de Student e correlação de Pearson, adotando-se $\mathrm{p}<0,05$ em todas as análises. A DMRA SU foi 2,3 $\pm 1,2 \mathrm{~cm}$ nas primíparas e 2,6 $\pm 1,4 \mathrm{~cm}$ nas

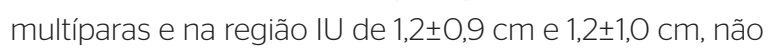
havendo diferença entre os grupos. A DMRA SU foi significativamente maior na IU tanto nas primíparas e multíparas ( $p<0,0001)$. Foi verificada associação significativa entre a DMRA SU e IU e entre DMRA SU, paridade e idade. Não foram observadas correlações da DMRA SU com o IMC e o TPP, e nenhuma correlação da DMRA IU com as variáveis estudadas. A DMRA SU foi similar entre os grupos e maior do que a IU. Encontrou-se correlação significativa entre DMRA SU e IU e, entre a DMRA SU com paridade e idade.
\end{abstract}

Descritores I período pós-parto; reto do abdome; fisioterapia; parto.

\begin{abstract}
I The present study compared the diastasis of rectus abdominis muscle (DRAM) above and below umbilicus in primiparous and multiparous and correlated these with maternal's age, number of births, body mass index (BMI) and labour. Women's submitted to a vaginal delivery were included: 100 primiparous women (aged $21.0 \pm 4.4$ years) and 100 multiparous women ( $27.2 \pm 6.2$ years). The DRAM was measurements $4.5 \mathrm{~cm}$ above and below de umbilical scar with a caliper. Statistical analysis was conducted by Student's t-test for dependent samples and Pearson's correlation, considering $p<0.05$. The DRAM above umbilicus in primiparous was $2.3 \pm 1.2 \mathrm{~cm}$ and $2.6 \pm 1.4 \mathrm{~cm}$ in multiparous and below umbilicus were $1.2 \pm 0.9 \mathrm{~cm}$ and $1.2 \pm 1.0$, showing no difference. The DRAM above umbilicus was higher than below in primiparous and multiparous ( $p<0.0001$ ). It was found a significant association between the DRAM above and below the umbilicus, and DRAM above umbilicus and maternal's age and number of births. No correlations with the BMI and labor were observed. DRAM above umbilicus was significantly greater in both groups, but similar when primiparous and multiparous were compared. A significant correlation was observed among DRAM above and below umbilicus, DRAM above umbilicus and maternal's age and number of births. Keywords I postpartum period; rectus abdominis; physical therapy specialty; parturition.
\end{abstract}

Estudo desenvolvido na Pontifícia Universidade Católica de Minas Gerais (PUC-Minas), em parceria com a Maternidade Pública Municipal de Betim - Betim (MG), Brasil.

'Fisioterapeuta; Doutora em Tocoginecologia pela Universidade Estadual de Campinas (UNICAMP) - Campinas (SP), Brasil; Professora Adjunta do curso de Fisioterapia da Universidade Federal de Sergipe (UFS) - Aracaju (SE), Brasil.

${ }^{2}$ Fisioterapeuta pela PUC-Minas - Betim (MG), Brasil; Especialista em Fisioterapia na Saúde da Mulher com aspectos na Dermatofuncional pela Faculdade Pitágoras - Belo Horizonte (MG), Brasil.

Endereço para correspondência: Dra. Mariana Tirolli Rett - Núcleo de Fisioterapia da Universidade Federal de Sergipe - Rua Claudio Batista s/n - Santo Antonio - CEP: 49060-100 - Aracaju (SE), Brasil - E-mail: marianatrb@gmail.com, maritrett@yahoo.com.br

Apresentação: jan. 2012 - Aceito para publicação: jun. 2012 - Fonte de financiamento: nenhuma - Conflito de interesse: nada a declarar - Parecer de aprovação no Comitê de Ética n० CAAE 0303.0.213.000-06. 


\section{INTRODUÇÃO}

O período após o parto, também conhecido como puerpério, é o momento quando as modificações locais e sistêmicas provocadas no organismo da mulher pela gravidez e parto retornam ao estado pré-gravídico ${ }^{1}$. É classificado em pós-parto imediato ( $1^{\circ}$ ao $\left.10^{\circ} \mathrm{dia}\right), \operatorname{tar}-$ dio ( $11^{\circ}$ ao $\left.45^{\circ} \mathrm{dia}\right)$ e remoto, que vai além dos 45 dias $^{1}$.

Durante a gestação ocorrem diversas adaptações fisiológicas ${ }^{2-4}$ e as alterações posturais podem contribuir para a anteversão pélvica acompanhada ou não de uma hiperlordose lombar ${ }^{4,5}$. Isso, associado ao crescimento uterino e estiramento da linha alba, podem interferir na biomecânica dos músculos retoabdominais.

As mudanças biomecânicas nos músculos abdominais facilitam o aparecimento da diástase dos músculos retoabdominais (DMRA), que pode ser definida como o afastamento entre estes dois músculos ${ }^{4,6-11}$. A etiologia da DMRA está relacionada às alterações hormonais (relaxina, estrógeno e progesterona) associadas à sobrecarga mecânica pelo crescimento uterino. Assim, todo o tecido conectivo local, incluindo a linha alba, ficam expostos, o que predispõe ao seu aparecimento ${ }^{2,7,10-15}$.

A tomografia computadorizada (TC) é considerada o padrão-ouro para avaliar a parede abdominal, mas esta expõe o paciente a radiações e possui alto custo ${ }^{16,17}$. A ultrassonografia (US) possui custo relativo, é uma técnica não invasiva, porém exige treinamento do técnico e a qualidade da imagem pode ser prejudicada pela interposição da gordura e fibrose ${ }^{17}$. Assim, de maneira mais prática, fisioterapeutas mensuram a DMRA nos períodos pré e pós-natal com as polpas digitais e estimam o afastamento dos músculos retoabdominais pela largura dos dedos. Entretanto, tal medida pode ser questionada, devido às variações individuais entre examinadores. Por isso, tem sido sugerida a utilização de um paquímetro, considerado um instrumento simples, de baixo custo e mais objetivo $0^{3,6,8,10,18}$.

Há controvérsias na literatura sobre o valor de referência da DMRA, podendo ser considerada como qualquer separação dos feixes musculares. Chiarello et $a .{ }^{11}$ a consideram como um valor $>2 \mathrm{~cm}$ acima da cicatriz umbilical e $<1 \mathrm{~cm}$ abaixo da cicatriz umbilical. Gilleard e Brown ${ }^{12}$ consideram como uma separação $>1 \mathrm{~cm}$ acima e abaixo do umbigo e $1,5 \mathrm{~cm}$ na cicatriz umbilical. Spitznagle, Leong e Van Dillen ${ }^{15}$ consideram a DMRA como $1 \mathrm{~cm}$ acima e abaixo do umbigo. Entretanto, Polden e Mantle ${ }^{4}$ consideram como a separação vertical de 2 a $3 \mathrm{~cm}$ de largura e de 12 a $15 \mathrm{~cm}$ de comprimento, podendo se estender por quase todo músculo retoabdominal.
Assim, dependendo do critério de avaliação, o afastamento destes músculos ocorre aproximadamente em $66 \%$ das gestantes no $3^{\circ}$ trimestre, podendo persistir de 30 a $60 \%$ no período de pós-parto ${ }^{10}$. Embora não haja consenso sobre a sua prevalência, duração e possíveis complicações a curto/longo prazo, alguns fatores podem estar relacionados à sua ocorrência, como: obesidade, multiparidade, gestações múltiplas, macrossomia fetal, poliidrâmnio, flacidez da musculatura abdominal pré-gravídica ${ }^{2,4}$. Contudo, poucos estudos correlacionam a DMRA com variáveis maternas e obstétricas, especialmente de puérperas submetidas exclusivamente ao parto vaginal.

A atuação do fisioterapeuta na área de obstetrícia demanda cada vez mais informações sobre a avaliação e identificação da DMRA, pois pode contribuir para o desenvolvimento de estratégias de prevenção e tratamento. Portanto, os objetivos do presente estudo foram avaliar e comparar a DMRA supraumbilical (SU) e infraumbilical (IU) entre primíparas e multíparas e correlacioná-la com a paridade, idade materna, o índice de massa corporal (IMC) e o tempo de trabalho de parto (TTP).

\section{METODOLOGIA}

Foi realizado um estudo observacional do tipo transversal no período de fevereiro a maio de 2008. O presente estudo foi aprovado pelo Comitê de Ética em Pesquisa da PUC-Minas (CAAE 0303.0.213.000-06) e foram atendidas as diretrizes da Resolução no 196/96 do Conselho Nacional de Saúde.

Foram incluídos dados de puérperas primíparas (somente um parto) e multíparas (no mínimo dois partos) submetidas exclusivamente ao parto vaginal e foi respeitado o mínimo de seis horas após o parto. Foram excluídos os dados daquelas que tivessem sido submetidas à cirurgias abdominais anteriores (cesárea, laparotomia exploradora, abdominoplastia), parto prematuro (antes de 37 semanas) e que a ficha estivesse incompleta. Para caracterização da amostra, foram coletadas informações provenientes dos prontuários, como: idade, estado civil, IMC, profissão, antecedentes obstétricos, incluindo: $\mathrm{pa}^{-}$ ridade, idade gestacional e TTP. Os dados foram coletados na Maternidade Pública Municipal de Betim, que é uma maternidade de baixo risco e referência de humanização na região metropolitana de Belo Horizonte.

A mensuração da DMRA foi realizada com as puérperas na posição supina com os quadris flexionados e 
joelhos fletidos a $90^{\circ}$, pés apoiados e membros superiores estendidos ao longo do corpo ${ }^{8,11,19}$. Em seguida, era solicitada uma flexão de tronco até que as espinhas das escápulas estivessem fora da superfície. Nesta posição, os dedos do avaliador foram introduzidos de maneira perpendicular às bordas mediais dos músculos retoabdominais e o espaço percebido entre os dedos era imediatamente medido com o com o paquímetro (Tramontina ${ }^{\circledR}$ ) - $150 \mathrm{~mm}$ e acurácia de $0,05 \mathrm{~mm}$. Os pontos de referência para avaliação foram demarcados com uma régua de $9 \mathrm{~cm}$, sendo o ponto central localizado na cicatriz umbilical (borda superior do umbigo) e as duas extremidades $4,5 \mathrm{~cm}$ acima e abaixo da cicatriz umbilical. A DMRA foi coletada por dois acadêmicos pesquisadores do curso de Fisioterapia da PUC-Minas, Betim, devidamente treinados pelo docente responsável ${ }^{19}$.

Para o cálculo amostral, foi utilizado o software Epi-Info 6.0. Nesta maternidade são realizados de 80 a 120 partos por mês e considerando os critérios de inclusão/exclusão e um universo de 350 mulheres, precisão desejada de 3\%, prevalência estimada de $66 \%{ }^{15}$ e nível de confiança de 95\%, a amostra estimada foi de 190 mulheres. Para análise descritiva da amostra, foram realizados média e desvio padrão, frequência e porcentagem. Foi aplicado o teste de normalidade de Kolmogorov-Smirnov e a partir da distribuição normal dos dados, adotaram-se testes paramétricos para as análises. $\mathrm{O}$ test $t$ de Student foi realizado para as variáveis independentes e para as correlações, o teste de correlação de Pearson, com nível de significância de $\mathrm{p}<0,05$, em todas as análises. Os dados finais foram informatizados e analisados com o programa estatístico Statistical Package for Social Science (SPSS), versão 13.0.

\section{RESULTADOS}

Foram selecionadas 237 fichas de dados, sendo incluídas 100 fichas de primíparas e 100 de multíparas com no mínimo 2 partos vaginais. As 37 fichas excluídas foram

Tabela 1. Características gerais e obstétricas das primíparas e multíparas

\begin{tabular}{lccc} 
& $\begin{array}{c}\text { Primíparas } \\
(\mathrm{n}=100)\end{array}$ & $\begin{array}{c}\text { Multíparas } \\
(\mathrm{n}=100)\end{array}$ & Valor $\mathrm{p}^{*}$ \\
\hline $\begin{array}{l}\text { Idade (anos) } \\
\text { Índice de massa } \\
\text { corporal }\left(\mathrm{kg} / \mathrm{m}^{2}\right)\end{array}$ & $21,0 \pm 4,4$ & $27,2 \pm 6,2$ & 0,001 \\
$\begin{array}{l}\text { Tempo de trabalho de } \\
\text { parto (minutos) }\end{array}$ & $343,4 \pm 213,3$ & $28,7 \pm 5,6$ & 0,002 \\
\hline
\end{tabular}

Valores apresentados como média đdesvio padrão; *teste $t$ de Student, $p<0,05$. por apresentarem antecedentes de partos cesáreos, parto prematuro e ficha de dados incompletas.

$\mathrm{Na}$ Tabela 1 observa-se que média de idade e do IMC foi significativamente maior no grupo das multíparas ( $p<0,0001$ e $p<0,002$, respectivamente). O TTP foi significativamente superior nas primíparas $(\mathrm{p}=0,015)$.

Entre as primíparas, 46 tinham parceiro fixo (casada/amasiada) e 44 eram solteiras. Com relação à ocupação, 59 eram do lar, 11 estudantes e as outras 30 tinham atividades distintas. Entre as multíparas, 86 tinham parceiro fixo (casada/amasiada) e 14 eram solteiras. Sessenta e sete puérperas eram do lar, 7 domésticas e as outras 26 tinham atividades distintas.

Observa-se, na Tabela 2, que DMRA SU foi $2,3 \pm 1,2 \mathrm{~cm}$ nas primíparas e $2,6 \pm 1,4 \mathrm{~cm}$ nas multíparas e na região IU de $1,2 \pm 0,9 \mathrm{~cm}$ e 1,2 $\pm 1,0 \mathrm{~cm}$, não havendo diferença significativa entre os grupos. Contudo, a DMRA SU foi significativamente maior do que a IU tanto nas primíparas $(p<0,0001)$ quanto nas multíparas $(p<0,0001)$.

Adicionalmente, foi verificada correlação significativa entre a DMRA SU e IU ( $\mathrm{p}=0,0001 ; \mathrm{r}=0,498)$, entre a DMRA SU e paridade ( $\mathrm{p}=0,0349 ; \mathrm{r}=0,161)$ e idade materna ( $p=0,0063 ; r=0,178)$. Não foram observadas correlações entre a DMRA SU e IMC ( $\mathrm{p}=0,257 ; \mathrm{r}=0,046)$ e TTP ( $p=0,164 ; r=0,033$ ). Também não foi observada correlação entre a DMRA IU e as variáveis maternas estudadas.

\section{DISCUSSÃO}

Este trabalho avaliou a DMRA de puérperas primíparas e multíparas submetidas exclusivamente a partos vaginais. A literatura ainda é divergente sobre qual afastamento é considerado DMRA, pois os autores utilizam critérios e instrumentos variados, o que dificulta a comunicação entre pesquisadores e as comparações clínicas. Segundo Berr et al. ${ }^{20}$ e Coldron et al..$^{21} \mathrm{em}$ nulíparas avaliadas com ultrassonografia, o afastamento na região $\mathrm{SU}$ foi de 1,3 e $1,1 \mathrm{~cm}$, respectivamente. Acredita-se que a DMRA do presente estudo seja

Tabela 2. Comparação da diástase dos músculos retoabdominais supra e infraumbilical entre primíparas e multíparas

\begin{tabular}{lccc} 
DMRA $(\mathrm{cm})$ & $\begin{array}{c}\text { Primíparas } \\
(\mathrm{n}=100)\end{array}$ & $\begin{array}{c}\text { Multiparas } \\
(\mathrm{n}=100)\end{array}$ & Valor $\mathbf{p}^{*}$ \\
\hline Supraumbilical & $2,3 \pm 1,2$ & $2,6 \pm 1,4$ & 0,109 \\
Infraumbilical & $1,2 \pm 0,9$ & $1,2 \pm 1,0$ & 0,843 \\
Valor $\mathrm{p}^{\star *}$ & 0,0001 & 0,0001 &
\end{tabular}

DMRA: diástase dos músculos retoabdominais; valores apresentados como média desvio padrão); teste $t$ de Student $p<0,05$, "primíparas vs. multíparas, **supraumbilical vs. infraumbilical 
superior (2,3 e 2,6 cm), por se tratar de primíparas e multíparas. Independentemente da paridade, o média da DMRA de puérperas está próximo aos encontrados por outros trabalhos nacionais, que avaliaram a DMRA tanto pelas polpas digitais quanto pelo paquímetro ${ }^{8,19}$.

Assim como achados anteriores ${ }^{19}$, a DMRA SU foi similar entre os grupos, embora nas multíparas tenha sido discretamente superior. Adicionalmente, observou-se correlação positiva entre a DMRA SU com a paridade e idade materna, assim como Souza, Oliveira e Lima ${ }^{22}$. Tais informações corroboram os achados de que mulheres com maior número de gestações e partos anteriores têm idade mais avançada e apresentam DMRA mais elevada ${ }^{7,11,14,15,22,23}$. Acredita-se que sucessivas gestações e partos possam contribuir para o estresse mecânico cumulativo do tecido conectivo da parede abdominal. Com o processo da gestação, a massa corporal e as dimensões do útero em constante aumento influenciam a morfologia musculoesquelética do tronco, aumentando as distâncias entre as inserções musculares o que gera prejuízo e desvantagem. Spitznagle, Leong e Van Dillen ${ }^{15}$ observaram que mulheres com idade superior a 50 anos, com maior número de gestações e de parto, apresentam uma maior prevalência de DMRA. Em relação à DMRA na região IU, não foi observada diferença entre os grupos. No entanto, de acordo com Chiarello et al. ${ }^{11}$ e Rett et al. ${ }^{19}$, a DMRA IU pode ser significativamente superior em multíparas.

Quanto às outras variáveis estudadas, embora o IMC das multíparas tenha sido significativamente maior, não foi observada correlação com a DMRA, conforme resultados anteriores ${ }^{19}$. Não houve correlação entre o TPP e a DMRA. Isso pode ser reforçado pelos resultados de Oliveira et al. ${ }^{24}$ que também não encontraram correlação da DMRA com o período expulsivo e encontraram correlação negativa entre a atividade eletromiográfica dos abdominais e a DMRA. Estes autores sugerem que a DMRA pode ser um parâmetro que influencia na geração de esforços voluntários durante o tempo do período expulsivo do parto, porém não deve ser considerada de forma isolada para o sucesso do andamento do trabalho de parto.

Foi observado que a média da DMRA foi significativamente menor na região IU, quando comparada com a região $\mathrm{SU}^{8,12,19}$, independentemente da paridade. Utilizando uma análise tridimensional, Gilleard e Brown $^{12}$ demonstraram valores de $6,0 \mathrm{~cm}$ na região $\mathrm{SU}$ e $3,2 \mathrm{~cm}$ na IU. Acredita-se que a DMRA seja menor na região IU pela diferença anatômica existente entre as disposições das fáscias que recobrem o retoabdominal.
A constituição da bainha do reto varia de acordo com o nível considerado na parede do abdome. A área de mudança está situada aproximadamente no meio da distância entre a cicatriz umbilical e a sínfise púbica e, geralmente, é marcada pela linha arqueada. Acima da linha arqueada, a bainha tem um folheto anterior (formado pela aponeurose de inserção do oblíquo externo e pela delaminação anterior da aponeurose do oblíquo interno) e um posterior (formado pela delaminação posterior da aponeurose do oblíquo interno e pela aponeurose de inserção do transverso) e abaixo da linha arqueada só existe o folheto anterior, formado pelas aponeuroses dos três músculos. Assim, na porção final desses músculos a disposição aponeurótica é diferente, evitando o afastamento ${ }^{25}$.

A avaliação da DMRA e o valor a ser considerado são especialmente importantes entre os fisioterapeutas, pois esses profissionais preocupam-se com a funcionalidade e integridade física dos indivíduos. Embora ainda não haja consenso, uma DMRA acima de 2,5 cm pode ser considerada prejudicial ${ }^{13,15}$, pois a partir de tal valor poderá interferir na capacidade da musculatura abdominal de estabilização do tronco, e em funções como postura, parto, defecação, respiração, parturição, movimentos do tronco, além da contenção visceral e estabilização lombar $7,9,11,12,14,26,27$. No presente estudo, a média de DMRA na região SU nas primíparas está próximo ao aceitável pela literatura, mas entre as multíparas, uma DMRA SU de 2,6 $\pm 1,4 \mathrm{~cm}$ pode indicar que são candidatas às disfunções provenientes da falta de estabilização abdominal e merecem atenção especial sobre a realização de exercícios abdominais no pós-parto. $\mathrm{O}$ prejuízo da estabilização abdominal pode predispor ao desenvolvimento de dor lombar e isso foi demonstrado por Toranto ${ }^{28}$ que após a redução cirúrgica da DMRA, obteve um alívio significativo da lombalgia.

Coldron et al. ${ }^{21}$ constataram que há uma redução parcial da DMRA até a oitava semana pós-parto, mas, quando avaliada um ano após o parto, não foi observado retorno aos valores pré-gravídicos. De acordo com Boissonnault e Blaschak ${ }^{14}$ nem todos os casos de DMRA são resolvidos espontaneamente após o parto. Isto justifica e reforça a necessidade de ser realizada a avaliação criteriosa da DMRA, para o planejamento adequado da conduta terapêutica. A realização de exercícios físicos devidamente supervisionados, tanto no período gestacional quanto no pós-parto, são importantes não só para tratamento de disfunções musculoesqueléticas, mas também para prevenção. Os exercícios de fortalecimento dos músculos abdominais priorizando 
o transverso do abdome têm apresentado sucesso. Chiarello et al. ${ }^{11}$ demonstraram que o grupo de gestantes submetidas a um protocolo de exercícios abdominais exibiu uma DMRA significativamente menor que as gestantes sedentárias. $\mathrm{O}$ mesmo foi observado em um estudo nacional no qual puérperas submetidas a um protocolo de exercícios abdominais reduziram significativamente a DMRA 18 horas após o parto vaginal ${ }^{8}$.

Para a prática clínica, a utilização de um paquímetro é mais recomendada do que a avaliação da DMRA com as polpas digitais ${ }^{10,11,18,28}$. Contudo, na necessidade de uma investigação mais minuciosa, os exames de imagem como ultrassom e tomografia podem ser necessários ${ }^{27}$. A utilização padronizada de um instrumento e pontos fixos a serem avaliados no abdome podem servir como referência para futuros estudos, além de possivelmente diminuir erros e subjetividade na forma de avaliação e interpretação dos dados. Avaliar adequadamente a DMRA é importante para orientar o funcionamento de serviços clínicos em obstetrícia e facilitar a comunicação entre pesquisadores e clínicos.

Este estudo apresenta algumas limitações, pois avaliou a DMRA apenas em puérperas submetidas ao parto vaginal, excluindo aquelas no pós-parto cesariano. Além disso, não correlacionou a DMRA com dados do recém-nascido, ganho de peso durante a gestação e não acompanhou estas mulheres nos pós-parto tardio e remoto. Delineamentos mais rigorosos, com maior tempo de seguimento e que identifiquem os fatores etiológicos, além das repercussões clínicas da DMRA serão importantes para profissionais engajados na assistência obstétrica e para a humanização do atendimento nas maternidades. Contudo, espera-se que os resultados do presente estudo possam contribuir para a elaboração de estratégias de prevenção e tratamento da DMRA e suas repercussões.

\section{CONCLUSÃO}

A DMRA supraumbilical e infraumbilical não apresentaram diferença significativa entre as primíparas e multíparas. Contudo, a DMRA supraumbilical foi significativamente maior do que a DMRA infraumbilical, independentemente da paridade. Encontrou-se correlação significativa entre DMRA supraumbilical e infraumbilical, paridade e idade materna. Não foram observadas correlações com IMC e TPP.

\section{REFERÊNCIAS}

1. Bacha CA, Rezende CAL. Puerpério fisiológico. In: Corrêa MD, editor. Noções práticas de obstetrícia. Rio de Janeiro: Medsi; 1999. p. 849-54.

2. Machado AV. Puerpério. In: Baracho E, editor. Fisioterapia aplicada à obstetrícia, uroginecologia e aspectos de mastologia. Rio de Janeiro: Guanabara Koogan; 2007. p. 225-40.

3. Lemos A, Caminha MA, Melo Junior EF, Andrade DA. Avaliação da força muscular respiratória no terceiro trimestre de gestação. Rev Bras Fisioter. 2005;9(2):151-6.

4. Polden M, Mantle J.. Fisioterapia em Ginecologia e Obstetrícia. $2^{\mathrm{a}}$ ed. São Paulo: Santos; 2002. O Período Pós-Natal; p. 223-73.

5. Kapandji AL. Fisiologia Articular: tronco e coluna vertebral. Rio de Janeiro: Guanabara Koogan; 2000. Músculos da parece abdominal: o oblíquo interno e o oblíquo externo; p. 100-1.

6. Bursch SG. Interrater reliability of diastasis recti abdominis measurement. Phys Ther. 1987;67(7):1077-9.

7. Lo T, Candido G, Janssen P. Diastasis of the recti abdominis in pregnancy: risk factors and treatment. Physiother Can. 1999;(3)32-37.

8. Mesquita LA, Machado AV, Andrade AV. Fisioterapia para redução da diástase dos músculos retos abdominais no pós-parto. Rev Bras Ginecol Obstet. 1999;21(5):267-72.

9. Strauhal MJ. Exercícios terapêuticos em obstetrícia. In: Hall CM, Brody LT. Exercício terapêutico na busca da função. Rio de Janeiro: Guanabara Koogan; 2001. p. 215-34.

10. Hsia M, Jones S. Natural resolution of rectus abdominis diastasis. Two single case studies. Aust J Physiother. 2000;46(4):301-7.

11. Chiarello CM, Falzone LA, McCaslin KE, Patel MN, Ulery KR. The effects of an exercise program on diastasis recti abdominis in pregnant women. Journal of Women's Health Physical Therapy. 2005:29(1):11-6.

12. Gilleard WL, Brown JM. Structure and function of the abdominal muscles in primigravid subjects during pregnancy and the immediate post birth period. Phys Ther. 1996;76(7):750-62.

13. Huge BS, Kisner C. Princípios de exercícios para a paciente obstétrica. In: Kisner C, Colby LA. Exercícios terapêuticos: fundamentos e técnicas. $4^{a}$ ed. São Paulo: Manole; 2005. p. 681-707.

14. Boissonnault JS, Blaschak MJ. Incidence of diastasis recti abdominis during the childbearing year. Phys Ther. 1988;68(7):1082-6.

15. Spitznagle TM, Leong FC, Van Dillen LR. Prevalence of diastasis recti abdominis in a urogynecological patient population. Int Urogynecol J Pelvic Floor Dysfunct. 2007;18(3):321-8.

16. Nahas FX. Ferreira LM, Augusto SM, Ghelfond C. Long-term follow-up correction of rectus diastasis. Plast Reconstr Surg. 2005;115(6):1736-1741.

17. Mendes DA, Nahas FX, Veiga DF, Mendes FV, Figueiras RG, Gomes $M C$, et al. Ultrasonography for measuring rectus abdominis muscles diastasis. Acta Cir Bras. 2007;22(3):182-6.

18. Boxer S, Jones S. Intra-rater reliability of rectus abdominis diastasis measurement using dial calipers. Aust J Physiother. 1997:43(2):109-14.

19. RettMT, Braga MD, Bernardes NO, AndradeSC. Prevalência de diástase dos músculos retoabdominais no puerpério imediato: comparação entre primíparas e multíparas. Rev Bras Fisioter. 2009:13(4):275-80.

20. Beer GM, Schuster A, Seifert B, Manestar M, Mihic-Probst D, Weber SA. The normal width of the linea alba in nulliparous women. Clin Anat. 2009:22(6):706-11

21. Coldron Y, Stokes MJ, Newham DJ, Cook K. Postpartum characteristics of rectus abdominis on ultrasound imaging. Man Ther. 2008;13:112-21. 
22. Souza CAAR, Oliveira RA, Lima ACG. Diástase dos músculos retos abdominais em puérperas na fase hospitalar. Fisioter Bras. 2009;10(5):333-38.

23. Turan V, Colluoglu C, Turkyilmaz E, Korucuoglu U. Prevalence of diastasis recti abdominis in the population of young multiparous adults in Turkey. Ginekol Pol. 2011;82(11):817-21.

24. Oliveira BD, Andrade AD, Lemos A, Brito VC, Pedrosa ML, Silva TN. Abdominal muscle electrical activity during labor expulsive stage: a cross-sectional study. Rev Bras Fisioter. 2011;15(6):445-51.
25. Moore KL. O abdome. In: Moore KL, editor. Anatomia orientada para a clínica. 3a ed. Rio de Janeiro: Guanabara Koogan; 1994. p. 117-23.

26. Sapsford R. Rehabilitation of pelvic floor muscles utilizing trunk stabilization. Man Ther. 2004;9(1):3-12.

27. Hodges PW. Is there a role for transversus abdominis in lumbo-pelvic stability? Man Ther. 1999;4(2):74-86.

28. Toranto IR. The relief of low back pain with the WARP abdominoplasty: a preliminary report. Plast Reconstr Surg. 1990;85(4):545-55. 